\title{
Superstability of derivations on Banach $*$-algebras
}

\section{Sun Young Jang ${ }^{*}$ (D)}

"Correspondence: jsym@ulsan.ac.kr Department of Mathematics, University of Ulsan, Ulsan, 44610, Korea

\begin{abstract}
In this paper, we show that approximate derivations on Banach $*$-algebras are exactly derivations and also show that approximate quadratic $*$-derivations on Banach *-algebras are exactly quadratic $*$-derivations by the fixed point theorem.
\end{abstract}

MSC: Primary 46S40; 39B52; 47H10; 39B62; 26E50; 47S40

Keywords: derivation; quadratic derivation; superstability; fixed point theorem

\section{Introduction}

Let $\mathcal{A}$ be a Banach $*$-algebra. A map $\delta: \mathcal{A} \rightarrow \mathcal{A}$ is called a derivation on $\mathcal{A}$ if it satisfies the following property:

$$
\begin{aligned}
& \delta(\lambda a+b)=\lambda \delta(a)+\delta(b), \\
& \delta(a b)=\delta(a) b+a \delta(b)
\end{aligned}
$$

for all $a, b \in \mathcal{A}$ and $\lambda \in \mathbf{C}$. Equation (1.2) is called the derivation property. If $\delta$ satisfies the additional condition $\delta\left(a^{*}\right)=\delta(a)^{*}$ for all $a \in \mathcal{A}$, then $\delta$ is called a $*$-derivation on $\mathcal{A}$. Sakai showed that if $\mathcal{A}$ is a $C^{*}$-algebra, then the $*$-derivation $\delta$ is bounded. And also he showed that $\delta(x)=a d_{i h}(x)=i(h x-x h)$ for some self-adjoint element $h$ in the enveloping von Neumann algebra $A^{\prime \prime}$ of the $C^{*}$-algebra A. If the self-adjoint element $h$ is in the multiplier algebra $M(\mathcal{A})$ of $\mathcal{A}, \delta$ is called an inner $*$-derivation on $\mathcal{A}$. Furthermore, if we put $U_{t}=\exp ^{i t h}$ for $h$ in $M(\mathcal{A})$ and $t \in \mathbf{R}$, then $U_{t}$ can be a unitary operator and generate a oneparameter group of $*$-automorphisms on $\mathcal{A}$. So bounded derivations of $C^{*}$-algebras have deep relations with generators of $C^{*}$-dynamical systems. Besides these, since derivations play an important role in the classifications of operator algebras, the theory of bounded *-derivations of $C^{*}$-algebras is very important in the theory of quantum mechanics and operator algebras [1-3].

We can say that a mathematical property is stable if any mathematical object satisfying a certain mathematical property approximately is near to the object exactly satisfying the mathematical property. On the stability of the functional equation, Ulam was the first beginner. He suggested the question 'Under what condition is there an additive mapping near an approximately additive mapping?' in 1940. Hyers [4] gave an affirmative answer for the problem of Ulam on the case of Banach spaces after one year. Since then, there

(c) The Author(s) 2017. This article is distributed under the terms of the Creative Commons Attribution 4.0 International License (http://creativecommons.org/licenses/by/4.0/), which permits unrestricted use, distribution, and reproduction in any medium, provided you give appropriate credit to the original author(s) and the source, provide a link to the Creative Commons license, and indicate if changes were made. 
have been a lot of results obtained related to the stability problems of various functional equations (for instances, [5-13]). In particular, some of the important functional equations are the following functional equations:

$$
\begin{aligned}
& f(x+y)=f(x)+f(y), \\
& f(x+y)+f(x-y)=2 f(x)+2 f(y)
\end{aligned}
$$

which are called Cauchy additive functional equation and Cauchy quadratic functional equation, respectively.

It is said that a mathematical property is superstable if every mathematical object satisfying approximately the property is an exact object satisfying it. The superstability phenomenon was first investigated by Baker, Lawrence and Zorzitto, etc. [14-17]. They showed the superstability of the exponential functional equation from the vector space to the set of real numbers. In the proof of the superstability of the exponential functional equation, the multiplicative property of the norm was the necessary condition. We say that when $E$ is a normed algebra and $\|x y\|=\|x\|\|y\|$ for all $x, y \in E$, the norm is multiplicative.

In this paper we define functional equations of a $*$-derivation and a quadratic $*$-derivation. And we show that the stability of $*$-derivations and $*$-quadratic derivations on Banach $*$-algebras without the multiplicative property of the norm are superstable by using the fixed point theorem.

In order to use the fixed point theory, we should introduce a fundamental result in the fixed point theory. Let $S$ be a set. A function $d: S \times S \rightarrow[0, \infty]$ is called a generalized metric on $S$ if $d$ satisfies

(1) $d(x, y)=0$ if and only if $x=y$;

(2) $d(x, y)=d(y, x)$ for all $x, y \in S$;

(3) $d(x, z) \leq d(x, y)+d(y, z)$ for all $x, y, z \in S$.

Theorem 1.1 $([18,19])$ Let $(S, d)$ be a complete generalized metric space, and let $J: S \rightarrow S$ be a strictly contractive mapping with Lipschitz constant $L<1$. Then, for each given element $x \in S$, either

$$
d\left(J^{n} x, J^{n+1} x\right)=\infty
$$

for all non-negative integers $n$ or there exists a positive integer $n_{0}$ such that

(1) $d\left(J^{n} x, J^{n+1} x\right)<\infty, \forall n \geq n_{0}$;

(2) the sequence $\left\{J^{n} x\right\}$ converges to a point $y^{*}$ in $S$;

(3) $J\left(y^{*}\right)=y^{*}$;

(4) $y^{*}$ is the unique fixed point of $J$ in the set $T=\left\{y \in S \mid d\left(J^{n_{0}} x, y\right)<\infty\right\}$;

(5) $d\left(y, y^{*}\right) \leq \frac{1}{1-L} d(y, J y)$ for all $y \in T$.

\section{Superstability of derivations on Banach $*$-algebras}

Let $\mathcal{A}$ denote a $*$-Banach algebra with the unit $e$ in Section 1 and Section 2.

Theorem 2.1 Let $\psi_{1}: \mathcal{A} \times \mathcal{A} \rightarrow[0, \infty)$ and $\psi_{2}: \mathcal{A} \rightarrow[0, \infty)$ be functions. Suppose that $f: \mathcal{A} \rightarrow \mathcal{A}$ is a mapping such that

$$
\|f(\lambda x+y)-\lambda f(x)-f(y)\| \leq \psi_{1}(x, y),
$$




$$
\begin{aligned}
& \|f(x y)-x f(y)-f(x) y\| \leq \psi_{1}(x, y), \\
& \left\|f\left(x^{*}\right)-f(x)^{*}\right\| \leq \psi_{2}(x)
\end{aligned}
$$

for all $\lambda \in \mathbb{T}$ and $x, y \in \mathcal{A}$. If there exist a natural number $s \in \mathbb{N}$ and $0<L<1$ such that $|s|^{-1} \psi_{1}(s x, s y)<L \psi_{1}(x, y),|s|^{-1} \psi_{1}(s x, y)<L \psi_{1}(x, y),|s|^{-1} \psi_{1}(x, s y)<L \psi_{1}(x, y)$, and $|s|^{-1} \psi_{2}(s x)<L \psi_{2}(x)$ for all $x, y \in \mathcal{A}$, then $f$ is a $*$-derivation on $\mathcal{A}$.

Proof If we put $x=y$ and $\lambda=1$ in (2.1), then we have

$$
\|f(2 x)-2 f(x)\| \leq \psi_{1}(x, x)
$$

for all $x \in \mathcal{A}$. We can see that

$$
\|f(n x)-n f(x)\| \leq \sum_{j=1}^{n-1} \psi_{1}(j x, x)
$$

for all $x, y \in \mathcal{A}$ and $n \geq 2$ by using the induction.

We put

$$
\Psi(x)=\sum_{j=1}^{s-1} \psi_{1}(j x, x)
$$

for $x \in \mathcal{A}$. Then we have

$$
\|f(s x)-s f(x)\| \leq \Psi(x)
$$

Let $S$ be the set of all functions $r: \mathcal{A} \rightarrow \mathcal{A}$. We define a function $d: S \times S \rightarrow[0, \infty]$ as follows:

$$
d(u, v)=\inf \{\alpha>0:\|u(x)-v(x)\| \leq \alpha \Psi(x), \forall x \in A\}
$$

for $u, v \in S$. We can easily show that $(S, d)$ is a generalized complete metric space. Define a function $H: S \rightarrow S$ by $H(u)(x)=s^{-1} u(s x)$. If we put

$$
d(u, v)=\alpha(u, v \in S)
$$

then we can have

$$
\|H(u)(x)-H(v)(x)\|=|s|^{-1}\|u(s x)-v(s x)\| \leq \alpha|s|^{-1} \Psi(s x) \leq L \alpha \Psi(x) .
$$

It follows that for $u, v \in S$

$$
d(H(u), H(v)) \leq L d(u, v) .
$$

Therefore $H$ is a strictly contractive mapping on $S$ with Lipschitz constant $L$. By (2.6),

$$
\|(H f)(x)-f(x)\|=\left\|s^{-1} f(s x)-f(x)\right\|=|s|^{-1}\|f(s x)-s f(x)\| \leq|s|^{-1} \Psi(x) .
$$


This means that $d(H(f), f) \leq 1 /|s|$. By Theorem 1.1, $H$ has a unique fixed point $h: \mathcal{A} \rightarrow \mathcal{A}$ in the set

$$
U=\{u \in S: d(u, H(f))<\infty\}
$$

We see that for each $x \in \mathcal{A}$

$$
h(x)=\lim _{m \rightarrow \infty} H^{m}(f(x))=\lim _{m \rightarrow \infty} s^{-m} f\left(s^{m} x\right) .
$$

From (2.6) we can have

$$
\begin{aligned}
& \|h(\lambda x+y)-\lambda h(x)-h(y)\| \\
& \quad=\lim _{n \rightarrow \infty}|s|^{-n}\left\|f\left(s^{n}(\lambda x+y)\right)-\lambda f\left(s^{n} x\right)-f\left(s^{n} y\right)\right\| \\
& \quad \leq \lim _{n \rightarrow \infty}|s|^{-n} \psi_{1}\left(s^{n} x, s^{n} y\right) \\
& \quad \leq \lim _{n \rightarrow \infty} L^{n} \psi_{1}(x, y)=0
\end{aligned}
$$

for all $x, y \in \mathcal{A}$ and $\lambda \in \mathbf{T}$. Next, let $\lambda=\lambda_{1}+\mathrm{i} \lambda_{2} \in \mathbb{C}$, where $\lambda_{1}, \lambda_{2} \in \mathbb{R}$. Let $\mu_{1}=\lambda_{1}-\left[\lambda_{1}\right]$ and $\mu_{2}=\lambda_{2}-\left[\lambda_{2}\right]$, where $[\lambda]$ denotes the integer part of $\lambda$. One can represent $\mu_{i}$ as $\mu_{i}=\frac{\lambda_{i, 1}+\lambda_{i, 2}}{2}$ such that $\lambda_{i, j} \in \mathbb{T}(1 \leq i, j \leq 2)$. Since we show that $h(\lambda x+y)=\lambda h(x)+h(y)$ for $\lambda \in \mathbf{T}$, we can infer that

$$
\begin{aligned}
h(\lambda x) & =h\left(\lambda_{1} x\right)+\mathrm{i} h\left(\lambda_{2} x\right) \\
& =\left(\left[\lambda_{1}\right] h(x)+h\left(\mu_{1} x\right)\right)+\mathrm{i}\left(\left[\lambda_{2}\right] h(x)+h\left(\mu_{2} x\right)\right) \\
& =\left(\left[\lambda_{1}\right] h(x)+\frac{1}{2} h\left(\lambda_{1,1} x+\lambda_{1,2} x\right)\right)+\mathrm{i}\left(\left[\lambda_{2}\right] h(x)+\frac{1}{2} h\left(\lambda_{2,1} x+\lambda_{2,2} x\right)\right) \\
& =\left(\left[\lambda_{1}\right] h(x)+\frac{1}{2} \lambda_{1,1} h(x)+\frac{1}{2} \lambda_{1,2} h(x)\right)+\mathrm{i}\left(\left[\lambda_{2}\right] h(x)+\frac{1}{2} \lambda_{2,1} h(x)+\frac{1}{2} \lambda_{2,2} h(x)\right) \\
& =\lambda_{1} h(x)+\mathrm{i} \lambda_{2} h(x) \\
& =\lambda h(x)
\end{aligned}
$$

for all $x \in \mathcal{A}$ and $\lambda \in \mathbb{C}$. So $h$ is a $\mathbb{C}$-linear map on $\mathcal{A}$. For the involution of $h$, we can have

$$
\begin{aligned}
\left\|h\left(x^{*}\right)-h(x)^{*}\right\| & =\lim _{n \rightarrow \infty}|s|^{-n}\left\|f\left(s^{n} x^{*}\right)-f\left(s^{n} x\right)^{*}\right\| \\
& \leq \lim _{n \rightarrow \infty}|s|^{-n} \psi_{2}\left(s^{n} x\right) \\
& \leq \lim _{n \rightarrow \infty} L^{n} \psi_{2}(x)=0 .
\end{aligned}
$$

Next, we are going to prove the derivation property of $h$. Replacing $x$ by $s^{n} x$ and $y$ by $s^{n} y$ in (2.2) and dividing by $s^{2 n}$, we get

$$
\left\|\frac{f\left(s^{n} x s^{n} y\right)}{s^{2 n}}-x \frac{f\left(s^{n} y\right)}{s^{n}}-\frac{f\left(s^{n} x\right)}{s^{n}} y\right\| \leq \frac{1}{|s|^{2 n}} \psi_{2}\left(s^{n} x, s^{n} y\right) \leq L^{2 n} \psi_{2}(x, y) .
$$


By taking $n \rightarrow \infty$, we have

$$
h(x y)=x h(y)+h(x) y
$$

for all $x, y \in \mathcal{A}$. It follows that $h$ is a $*$-derivation on $\mathcal{A}$. Next, if we replace $x$ by $s^{n} x$ in (2.2) and divide by $s^{n}$, we get

$$
\left\|\frac{f\left(s^{n} x y\right)}{s^{n}}-x f(y)-\frac{f\left(s^{n} x\right)}{s^{n}} y\right\| \leq \frac{1}{|s|^{n}} \psi_{2}\left(s^{n} x, y\right) \leq L^{n} \psi_{2}(x, y)
$$

for all $x, y \in \mathcal{A}$ and all $n \in \mathbb{N}$. By taking $n \rightarrow \infty$, we have

$$
h(x y)=x f(y)+h(x) y
$$

for all $x, y \in \mathcal{A}$. Fix $m \in \mathbb{N}$. And considering the following equations

$$
\begin{aligned}
x f\left(s^{m} y\right) & =h\left(s^{m} x y\right)-h(x) s^{m} y \\
& =s^{m} x f(y)
\end{aligned}
$$

for all $x, y \in \mathcal{A}$, we can get $x f(y)=x \frac{f\left(s^{m} y\right)}{s^{m}}$ for all $x, y \in \mathcal{A}$ and each $m \in \mathbb{N}$. By taking $m \rightarrow \infty$, we have $x f(y)=x h(y)$. If we put $x=e$, then $h(y)=f(y)$ for all $y \in \mathcal{A}$. So $f$ is an exactly $*-$ derivation on $\mathcal{A}$.

\section{Superstability of quadratic $*$-derivations on Banach $*$-algebras}

Definition 3.1 A mapping $\delta: \mathcal{A} \rightarrow \mathcal{A}$ is a $*$-quadratic derivation of $\mathcal{A}$ if a map $\delta$ satisfies the following properties: for all $a, b \in \mathcal{A}$ and $\lambda \in \mathbb{C}$,

(1) $\delta(a+b)+\delta(a-b)-2 \delta(a)-2 \delta(b)=0$;

(2) $\delta$ is quadratic homogeneous, that is, $\delta(\lambda a)=\lambda^{2} \delta(a)$;

(3) $\delta(a b)=\delta(a) b^{2}+a^{2} \delta(b)$;

(4) $\delta\left(a^{*}\right)=\delta(a)^{*}$.

Theorem 3.2 Let $\psi_{1}: \mathcal{A} \times \mathcal{A} \rightarrow[0, \infty)$ and $\psi_{2}: \mathcal{A} \rightarrow[0, \infty)$ be functions. Suppose that $f: \mathcal{A} \rightarrow \mathcal{A}$ is a mapping such that

$$
\begin{aligned}
& \|f(x+y)+f(x-y)-2 f(x)-2 f(y)\| \leq \psi_{1}(x, y), \\
& \left\|f(x y)-x^{2} f(y)-f(x) y^{2}\right\| \leq \psi_{1}(x, y) \\
& \left\|f(\lambda x)-\lambda^{2} f(x)\right\| \leq \psi_{2}(x) \\
& \left\|f\left(x^{*}\right)-f(x)^{*}\right\| \leq \psi_{2}(x)
\end{aligned}
$$

for all $\lambda \in \mathbb{C}$ and $x, y \in \mathcal{A}$. If there exist a natural number $s \in \mathbb{N}$ and $0<L<1$ such that $2^{-2 s} \psi_{1}\left(2^{s} x, 2^{s} y\right)<L \psi_{1}(x, y), 2^{-2 s} \psi_{1}\left(2^{s} x, y\right)<L \psi_{1}(x, y), 2^{-2 s} \psi_{1}\left(x, 2^{s} y\right)<L \psi_{1}(x, y)$, and $2^{-2 s} \psi_{2}\left(2^{s} x\right)<L \psi_{2}(x)$ for all $x, y \in \mathcal{A}$, then $f$ is a $*$-quadratic derivation on $\mathcal{A}$.

Proof If we put $x=y$ and $\lambda=1$ in (3.1), then we have

$$
\|f(2 x)-4 f(x)\| \leq \psi_{1}(x, x), \quad x \in \mathcal{A}
$$


By induction on $n$, we can see that

$$
\left\|f\left(2^{n} x\right)-2^{2 n} f(x)\right\| \leq \sum_{i=0}^{n-1} 2^{2(n-i)} \psi_{1}\left(2^{i} x, 2^{i} x\right)
$$

for all $x, y \in \mathcal{A}$ and $n \geq 2$. For simplicity, if we put

$$
\Psi(x)=\sum_{i=0}^{s-1} 2^{2(s-i)} \psi_{1}\left(2^{i} x, 2^{i} x\right)
$$

then we have

$$
\left\|f\left(2^{s} x\right)-2^{2 s} f(x)\right\| \leq \Psi(x) .
$$

Let $S$ be the set of all functions $u: \mathcal{A} \rightarrow \mathcal{A}$. We define a function $d: S \times S \rightarrow[0, \infty]$ as follows:

$$
d(u, v)=\inf \{\alpha>0:\|u(x)-v(x)\| \leq \alpha \Psi(x), \forall x \in \mathcal{A}\}
$$

We can easily show that $(S, d)$ is a generalized complete metric space. Define a function $H: S \rightarrow S$ by $H(u)(x)=2^{-2 s} u\left(2^{s} x\right)$. If we put $d(u-v)=\alpha$ for $u, v \in S$, then we can have

$$
\|H(u)(x)-H(v)(x)\|=2^{-2 s}\left\|u\left(2^{s} x\right)-v\left(2^{s} x\right)\right\| \leq \alpha 2^{-2 s} \Psi\left(2^{s} x\right) \leq L \alpha \Psi(x) .
$$

It follows that for $u, v \in S$

$$
d(H(u), H(v)) \leq L d(u, v) .
$$

Hence $\mathrm{H}$ is a strictly contractive mapping on $X$ with Lipschitz constant $L$. We have that for $x \in \mathcal{A}$

$$
\|(H f)(x)-f(x)\|=\left\|2^{-2 s} f\left(2^{s} x\right)-f(x)\right\|=2^{-2 s}\left\|f\left(2^{s}\right)-2^{2 s} f(x)\right\| \leq 2^{-2 s} \Psi(x) .
$$

This means that $d(H(f), f) \leq 1 / 2^{2 s}$. By Theorem $1.1, H$ has a unique fixed point $h: \mathcal{A} \rightarrow A$ in the set

$$
U=\{u \in X: d(u, H(f))<\infty\}
$$

and for each $x \in \mathcal{A}$

$$
h(x)=\lim _{m \rightarrow \infty} H^{m}(f(x))=\lim 2^{-2 s m} f\left(2^{s m} x\right) .
$$

From (3.9), we can have

$$
\begin{aligned}
\|h(x+y)+h(x-y)-2 h(x)-2 h(y)\| \\
\quad=\lim _{n \rightarrow \infty} 2^{-2 s n} \| f\left(2^{s n}(x+y)+f\left(2^{s n}(x-y)\right)-2 f\left(2^{s n} x\right)-2 f\left(2^{s n} y\right) \|\right.
\end{aligned}
$$




$$
\begin{aligned}
& \leq \lim _{n \rightarrow \infty} 2^{-2 n s} \psi_{1}\left(2^{n s} x, 2^{n s} y\right) \\
& \leq \lim _{n \rightarrow \infty} L^{n} \psi_{1}(x, y)=0
\end{aligned}
$$

for all $x, y \in \mathcal{A}$. So $h$ is a quadratic map on $\mathcal{A}$. Since we can have that

$$
\begin{aligned}
& \left\|h(\lambda x)-\lambda^{2} h(x)\right\| \\
& =\lim _{n \rightarrow \infty} 2^{-2 n s}\left\|f\left(2^{n s}(\lambda x)\right)-\lambda^{2} f\left(2^{n s} x\right)\right\| \\
& \leq \lim _{n \rightarrow \infty} 2^{-2 n s} \psi_{2}\left(2^{n s} x\right) \\
& \leq \lim _{n \rightarrow \infty} L^{n} \psi_{2}(x)=0,
\end{aligned}
$$

it follows that $h$ is quadratic homogeneous.

Next, if we replace $x$ by $2^{n s} x$ in (3.2) and divide by $2^{-2 s n}$, then we get

$$
\begin{aligned}
\left\|\frac{f\left(2^{n s} x y\right)}{2^{2 n s}}-x^{2} f(y)-\frac{f\left(2^{n s} x\right)}{2^{2 n s}} y^{2}\right\| & \leq \frac{1}{2^{2 n s}} \psi_{1}\left(2^{n s} x, y\right) \\
& \leq L^{n} \psi_{1}(x, y)
\end{aligned}
$$

for all $x, y \in \mathcal{A}$ and all $n \in \mathbb{N}$. By taking $n \rightarrow \infty$, we have

$$
h(x y)=x^{2} f(y)+h(x) y^{2}
$$

for all $x, y \in \mathcal{A}$. Fix $m \in \mathbb{N}$. If we consider the following equations

$$
\begin{aligned}
x^{2} f\left(2^{m s} y\right) & =h\left(2^{m s} x y\right)-h\left(2^{m s} x\right) y^{2} \\
& =2^{2 m s} x^{2} f(y)+h\left(2^{m s} x\right) y^{2}-h\left(2^{m s} x\right) y^{2} \\
& =2^{2 m s} x^{2} f(y)
\end{aligned}
$$

for all $x, y \in \mathcal{A}$, then we have $x^{2} f(y)=x^{2} \frac{f\left(2^{m s} y\right)}{2^{2 m s}}$ for all $x, y \in A$ and for each $m \in \mathbb{N}$. Taking $m \rightarrow \infty$, we have $x^{2} f(y)=x^{2} h(y)$. If we put $x=e$, then $h(y)=f(y)$ for all $y \in \mathcal{A}$. So $f$ is a *-quadratic derivation on $\mathcal{A}$.

\section{Derivations on $C^{*}$-ternary algebras}

A $C^{*}$-ternary algebra is a complex Banach space $\mathcal{A}$ equipped with a ternary product $(x, y, z) \mapsto[x, y, z]$ of $\mathcal{A}^{3}$ into $\mathcal{A}$ satisfying the following properties:

(1) $[\lambda x+u, y, z]=\lambda[x, y, z]+[u, y, z]$ for all $\lambda \in \mathbb{C}$;

(2) $[x, \lambda y+u, z]=\bar{\lambda}[x, y, z]+[x, u, z]$ for all $\lambda \in \mathbb{C}$;

(3) $[x, y, \lambda z+u]=\lambda[x, y, z]+[x, y, u]$ for all $\lambda \in \mathbb{C}$;

(4) $[x, y,[z, w, v]]=[x,[w, z, y], v]=[[x, y, z], w, v]$;

(5) $\|[x, y, z]\| \leq\|x\| \cdot\|y\| \cdot\|z\|$;

(6) $\|[x, x, x]\|=\|x\|^{3}$ (see $\left.[20,21]\right)$

for $x, y, z, u, v, w \in \mathcal{A}$. We say that a $C^{*}$-ternary algebra $\mathcal{A}$ has the unit $e$ if there exists a unique element $e \in \mathcal{A}$ such that $x=[x, e, e]=[e, e, x]$ for all $x \in \mathcal{A}$. Also we can define an 
involution $*$ on the $C^{*}$-ternary algebra $\mathcal{A}$ with the unit $e$ such as $[e, x, e]=x^{*}$ for each $x \in \mathcal{A}$. A mapping $\delta: \mathcal{A} \rightarrow \mathcal{A}$ is called a $C^{*}$-ternary derivation if it satisfies

$$
\begin{aligned}
& \delta([x, y, z])=[\delta(x), y, z]+[x, \delta(y), z]+[x, y, \delta(z)], \\
& \delta(\lambda x+y)=\lambda \delta(x)+\delta(y)
\end{aligned}
$$

for all $x, y, z \in \mathcal{A}$ and $\lambda \in \mathbb{C}$. In addition, if $\delta$ satisfies that $\delta([e, x, e])=[e, \delta(x), e]$ on the $C^{*}$ ternary algebra $\mathcal{A}$ with the unit $e$, then it is said that $\delta$ is an involutive $C^{*}$-ternary derivation on $A$.

Theorem 4.1 Let $\mathcal{A}$ be a $C^{*}$-ternary algebra with the unit e. Let $\psi_{1}: \mathcal{A}^{2} \rightarrow[0, \infty)$ and $\psi_{2}: \mathcal{A}^{3} \rightarrow[0, \infty)$ be functions. Suppose that $f: \mathcal{A} \rightarrow \mathcal{A}$ is a mapping such that

$$
\begin{aligned}
& \|f(\lambda x+y)-\lambda f(x)-f(y)\| \leq \psi_{1}(x, y), \\
& \|f([x, y, z])-[f(x), y, z]-[x, f(y), z]-[x, y, f(z)]\| \leq \psi_{2}(x, y, z), \\
& \|f([e, y, e])-[e, f(y), e]\| \leq \psi_{2}(e, y, e)
\end{aligned}
$$

for all $\lambda \in \mathbb{T}$. Also suppose that there exist a natural number $s \in \mathbb{N}$ and $0<L<1$ such that $|s|^{-(i+j)} \psi_{1}\left(s^{i} x, s^{j} y\right)<L^{i+j} \psi_{1}(x, y),|s|^{-(i+j+k)} \psi_{2}\left({ }^{i} s x, s^{j} y, s^{k} z\right)<L^{i+j+k} \psi_{2}(x, y, z)$ for all $x, y, z \in \mathcal{A}$ and $i, j, k=0,1$. Then $f$ is an involutive $C^{*}$-ternary derivation on $\mathcal{A}$.

Proof If we put

$$
\Psi(x)=\sum_{j=1}^{s-1} \psi_{1}(j x, x)
$$

for $x \in \mathcal{A}$, then we have

$$
\|f(s x)-s f(x)\| \leq \Psi(x)
$$

similar to Theorem 2.1. Let $S$ be the set of all functions $r: \mathcal{A} \rightarrow \mathcal{A}$. We define a function $d: S \times S \rightarrow[0, \infty]$ as follows:

$$
d(u, v)=\inf \{\alpha>0:\|u(x)-v(x)\| \leq \alpha \Psi(x), \forall x \in A\}
$$

for $u, v \in S$. We can easily show that $(S, d)$ is a generalized complete metric space. Define a function $H: S \rightarrow S$ by $H(u)(x)=s^{-1} u(s x)$. When

$$
d(u, v)=\alpha(u, v \in S),
$$

then we can have

$$
\|H(u)(x)-H(v)(x)\|=|s|^{-1}\|u(s x)-v(s x)\| \leq \alpha|s|^{-1} \Psi(s x) \leq L \alpha \Psi(x) .
$$

It follows that for $u, v \in S$

$$
d(H(u), H(v)) \leq L d(u, v) .
$$


Therefore, $H$ is a strictly contractive mapping on $S$ with Lipschitz constant $L$. By (4.4),

$$
\|(H f)(x)-f(x)\|=\left\|s^{-1} f(s x)-f(x)\right\|=|s|^{-1}\|f(s x)-s f(x)\| \leq|s|^{-1} \Psi(x) .
$$

This means that $d(H(f), f) \leq 1 /|s|$. By Theorem 1.1, $H$ has a unique fixed point $h: \mathcal{A} \rightarrow \mathcal{A}$ in the set

$$
U=\{u \in S: d(u, H(f))<\infty\} .
$$

We see that for each $x \in \mathcal{A}$

$$
h(x)=\lim _{m \rightarrow \infty} H^{m}(f(x))=\lim _{m \rightarrow \infty} s^{-m} f\left(s^{m} x\right) .
$$

We can see that $h$ is a $\mathbb{C}$-linear map on $\mathcal{A}$ similar to the proof of Theorem 2.1. Now we are going to prove the $C^{*}$-ternary derivation property of $h$.

$$
\begin{aligned}
& \|h([x, y, z])-[h(x), y, z]-[x, h(y), z]-[x, y, h(z)]\| \\
& \quad=\lim _{n \rightarrow \infty}|s|^{-3 n}\left\|f\left(s^{3 n}[x, y, z]\right)-s^{2 n}\left[f\left(s^{n} x\right), y, z\right]-s^{2 n}\left[x, f\left(s^{n} y\right), z\right]-s^{2 n}\left[x, y, f\left(s^{n} z\right)\right]\right\| \\
& \quad \leq \lim _{n \rightarrow \infty}|s|^{-3 n} \psi_{1}\left(s^{n} x, s^{n} y, s^{n} z\right) \\
& \quad \leq \lim _{n \rightarrow \infty} L^{3 n} \psi_{1}(x, y, z)=0 .
\end{aligned}
$$

Hence we have

$$
h([x, y, z])=[h(x), y, z]+[x, h(y), z]+[x, y, h(z)]
$$

for all $x, y, z \in \mathcal{A}$. For the involution of $h$, we can have

$$
\begin{aligned}
& \|h([e, x, e])-[e, h(x), e]\| \\
& \quad=\lim _{n \rightarrow \infty}|s|^{-3 n}\left\|f\left(s^{3 n}[e, x, e]\right)-s^{2 n}\left[e, f\left(s^{n} x\right), e\right]\right\| \\
& \quad \leq \lim _{n \rightarrow \infty}|s|^{-3 n} \psi_{1}\left(s^{n} e, s^{n} x, s^{n} e\right) \\
& \quad \leq \lim _{n \rightarrow \infty} L^{3 n} \psi_{1}(e, x, e)=0 .
\end{aligned}
$$

So $h$ is an involutive $C^{*}$-ternary derivation on $\mathcal{A}$.

Replacing $y$ by $s^{n} y$ and $z$ by $s^{n} z$ in (4.2), dividing by $s^{2 n}$, and letting $n$ go to the infinity, we get

$$
\begin{aligned}
& \lim _{n \rightarrow \infty}\left\|s^{-2 n}\left(f\left(\left[x, s^{n} y, s^{n} z\right]\right)-\left[f(x), s^{n} y, s^{n} z\right]-s^{n}\left[x, f\left(s^{n} y\right), z\right]-s^{n}\left[x, y, f\left(s^{n} z\right)\right]\right)\right\| \\
& \quad=\lim _{n \rightarrow \infty}|s|^{-2 n}\left(\left\|f\left(s^{2 n}[x, y, z]\right)-s^{2 n}[f(x), y, z]-s^{n}\left[x, f\left(s^{n} y\right), z\right]-s^{n}\left[x, y, f\left(s^{n} z\right)\right]\right\|\right) \\
& \quad \leq \lim _{n \rightarrow \infty}|s|^{-2 n} \psi_{1}\left(x, s^{n} y, s^{n} z\right) \\
& \quad \leq \lim _{n \rightarrow \infty} L^{2 n} \psi_{1}(x, y, z)=0 .
\end{aligned}
$$


So we have

$$
h([x, y, z])=[f(x), y, z]+[x, h(y), z]+[x, y, h(z)]
$$

for all $x, y, z \in \mathcal{A}$. In (4.7) and (4.8) we put $f(x)-h(x)$ instead of $y$ and $z$, then we can get $\|h(x)-f(x)\|=0$. So $f$ is an exactly involutive $C^{*}$-ternary derivation on $\mathcal{A}$.

\section{Acknowledgements}

This paper was partially supported by the Research Fund of University of Ulsan, 2017.

The author would like to thank the referees for their useful comments.

\section{Competing interests}

The author declares that she has no competing interests.

\section{Author's contributions}

The author conceived of the study, participated in its design and coordination, drafted the manuscript, participated in the sequence alignment, and read and approved the final manuscript.

\section{Publisher's Note}

Springer Nature remains neutral with regard to jurisdictional claims in published maps and institutional affiliations.

Received: 3 January 2017 Accepted: 15 June 2017 Published online: 05 July 2017

\section{References}

1. Bratteli, O: Derivation, Dissipation and Group Actions on C*-Algebras. Lecture Notes in Math., vol. 1229. Springer, Berlin (1986)

2. Bratteli, O, Kishimoto, A, Robinson, DW: Approximately inner derivations. Math. Scand. 103, 141-160 (2008)

3. Jang, SY: Approximate $*$-derivations on fuzzy Banach $*$-algebras. Adv. Differ. Equ. doi:10.1180/1687-1847-2012-132

4. Hyers, DH: On the stability of the linear functional equation. Proc. Natl. Acad. Sci. USA 27, $222-224$ (1941)

5. Czerwik, S: On the stability of the quadratic mapping in normed spaces. Abh. Math. Semin. Univ. Hamb. 62, 59-64 (1992)

6. Cădariu, L, Găvruta, L, Găvruta, P: On the stability of an affine functional equation. J. Nonlinear Sci. Appl. 6, 60-67 (2013)

7. Hyers, DH, Isac, G, Rassias, TM: Stability of Functional Equations in Several Variables. Birkhäuser, Basel (1998)

8. Jung, SM, Popa, D, Rassias, MT: On the stability of the linear functional equation in a single variable on complete metric groups. J. Glob. Optim. 59, 165-171 (2014)

9. Kannappan, P: Functional Equations and Inequalities with Applications. Springer, Berlin (2009)

10. Miheţ, D, Radu, V: On the stability of the additive Cauchy functional equation in random normed spaces. J. Math. Anal. Appl. 343, 567-572 (2008)

11. Moslehian, MS, Rahbarnia, F, Sahoo, PK: Approximate double centralizers are exact double centralizers. Bol. Soc. Mat. Mexicana 13, 111-122 (2007)

12. Skof, F: Proriet locali e approssimazione di operatori. Rend. Semin. Mat. Fis. Milano 53, 113-129 (1983)

13. Rassias, TM: On the stability of the linear mapping in Banach spaces. Proc. Am. Math. Soc. 72, $297-300$ (1978)

14. Baker, JA, Lawrence, J, Zorzitto, F: The stability of the equation $f(x+y)=f(x) f(y)$. Proc. Am. Math. Soc. 74, 242-246 (1979)

15. Baker, JA: The stability of the cosine equation. Proc. Am. Math. Soc. 80, 411-416 (1980)

16. Cholewa, PW: The stability of the sine equation. Proc. Am. Math. Soc. 88, 631-634 (1983)

17. Szèkelyhidi, L: The stability of the sine and cosine functional equations. Proc. Am. Math. Soc. 110, 109-115 (1990)

18. Cădariu, L, Radu, V: Fixed points and the stability of Jensen's functional equation. J. Inequal. Pure Appl. Math. 4(1), Article ID 4 (2003)

19. Diaz, J, Margolis, B: A fixed point theorem of the alternative for contractions on a generalized complete metric space. Bull. Am. Math. Soc. 74, 305-309 (1968)

20. Amyari, M, Moslehian, MS: Approximately ternary semigroup homomorphisms. Lett. Math. Phys. 77, 1-9 (2006)

21. Zettl, H: A characterization of ternary rings of operators. Adv. Math. 48, 117-143 (1983) 